\title{
Particle dynamics in the stellar magnetosphere by gravitational collapse
}

\author{
Volodymyr Kryvdyk ${ }^{1}$ \\ National Taras Shevchenko University of Kyiv, 64, Volodymyrs'ka St.,01601 Kyiv, Ukraine \\ email: kryvdyk@univ.kiev.ua
}

\begin{abstract}
The particle dynamics and in the stellar magnetosphere during gravitational collapse is investigated. The formations of relativistic jets and the generation of the radiation bursts in the stellar magnetosphere by gravitational collapse are considered. As follows from results, the stars on the stage of gravitational collapse must be powerful sources of the relativistic jets and the non-thermal radiation. These jets will formed in the polar caps of collapsing stars magnetospheres, when the stellar magnetic field increases during collapse and the charged particles will be accelerate. These jets will generate the non-thermal radiation. The radiation flux grows with decreasing stellar radius and can be observed in the form of radiation burst in wide band wave- from radio to gamma-ray. These bursts radiation can be observed as gamma- and X- rays bursts.
\end{abstract}

Keywords. gravitational collapse, stellar magnetosphere, gamma- and X-rays bursts

\section{Particles acceleration and their nonthermal emission in magnetosphere}

Particles acceleration and generation of electromagnetic radiation in magnetosphere to result from processes Kryvdyk (1999), Kryvdyk (2004), Kryvdyk \& Agapitov (2005a), and Kryvdyk \& Agapitov (2005b) 1) Magnetic fields of collapsing stars increase during the collapse. 2) These variable magnetic fields accelerate the charged particles in the magnetospheres. 3) These particles emit the electromagnetic waves in the wide frequency band, from gamma rays to radio waves. 4)The radiation flux grows during collapse and to reaches a maximum on the final stage of collapse. This radiation can be observed as bursts in the all-frequency band, from radio to gamma ray. The burst duration completes with the collapse time. The radiation flux from collapsing on the final stage of collapse exceeds the initial flux a million times. 5) Thus the collapsing stars can be the powerful sources of the non-thermal radiation when before a supernova flares, the star compress and emit bursts.

Model particles generation in magnetosphere of collapsing stars give on figure 1.

\section{Conclusion}

1.Charged particles will accelerate in the magnetosphere of collapsing star to relativistic energy. 2. Relativistic polar jets will formed during collapse (figure 2). 3 . These jets emit the electromagnetic radiation in all frequency bands. 4 . This radiation can be observed as bursts from radio to gamma ray. 


\begin{tabular}{|c|c|c|}
\hline \multicolumn{2}{|c|}{$\begin{array}{l}\text { Acceleration of the initial protons and electrons } \\
\text { in magnetosphere during collapse }\end{array}$} & \\
\hline & \begin{tabular}{l|l}
1 \\
\end{tabular} & \\
\hline $\begin{array}{l}\text { Generation and acceleration } \\
\text { secondary particles }\end{array}$ & $\begin{array}{c}\text { Generation and acceleration } \\
\text { third particles }\end{array}$ & $\begin{array}{c}\text {... multiple generation } \\
\text { and acceleration third } \\
\text { particles }\end{array}$ \\
\hline Secondary particles & Third particles & Multiple particles \\
\hline $\begin{array}{l}\text { Protons (p) } \rightarrow \\
\text { Neutrons (n) } \\
\text { Electrons }\left(\mathrm{e}^{-}\right) \\
\text {Positrons }\left(\mathrm{e}^{+}\right) \\
\text {Mesons }(\pi, \mu) \\
\begin{array}{l}\text { Neutrinos }(v) \\
\text { Fotons }(\gamma)\end{array}\end{array}$ & $\begin{array}{l}\text { Protons (p) } \rightarrow \\
\text { Neutrons (n) } \\
\text { Electrons }\left(\mathrm{e}^{-}\right) \\
\text {Positrons }\left(\mathrm{e}^{+}\right) \\
\text {Mesons }(\pi, \mu) \\
\begin{array}{l}\text { Neutrinos }(\mathrm{v}) \\
\text { Fotons }(\gamma)\end{array}\end{array}$ & $\begin{array}{c}\text { Protons (p) } \rightarrow \\
\text { Neutrons (n) } \\
\text { Electrons }\left(\mathrm{e}^{-}\right) \\
\text {Positrons }\left(\mathrm{e}^{+}\right) \\
\text {Mesons }(\pi, \mu) \\
\begin{array}{c}\text { Neutrinos }(\mathrm{v}) \\
\text { Fotons }(\gamma)\end{array}\end{array}$ \\
\hline
\end{tabular}

Figure 1. Model particle generation in magnetosphere.

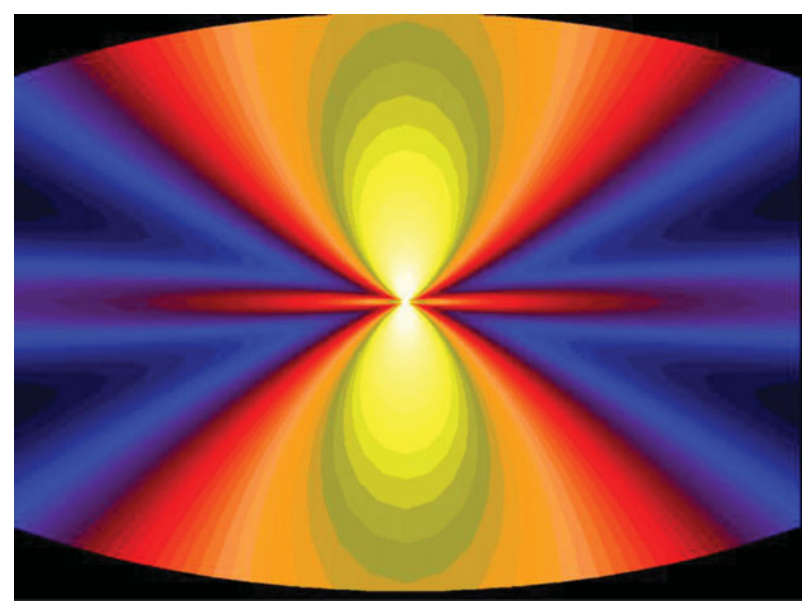

Figure 2. Jets in magnetosphere of collapsing stars

\section{References}

Kryvdyk, V. 1999, MRAS, 309, 593

Kryvdyk, V. 2004, Adv. Sp. Res., 33, 484

Kryvdyk, V. \& Agapitov, A. 2005a, ASP Conf. Ser., 334, 277

Kryvdyk, V. \& Agapitov, A. 2005b, Adv. Sp. Res., 330, 415

Kryvdyk, V. 2005, Springer Proc. Phys., 99, 215 